\title{
Summability Methods, the Strong Asymptotic Condition, and Unitarity in Quantum Field Theory*
}

\author{
Barry Simon $\dagger$ \\ Departments of Mathematics and Physics, Princeton University, Princeton, New Jersey 08540
}

(Received 28 February 1972)

\begin{abstract}
We discuss a summability mechanism which preserves nonlinear perturbative conditions such as unitarity of the Feynman series. This condition, which relates a function $f(z)$ and a series $\sum a_{n} z^{n}$ by the requirement$$
\left|f(z)-\sum_{n=0}^{N} a_{n} z^{n}\right| \leqslant A \sigma^{N+1}(N+1) !|z|^{N+1},
$$

is applicable to certain divergent series.

It has been known for some time that the perturbation series in super-renormalizable selfcoupled boson field theories diverge, ${ }^{1}$ and there have been a variety of mechanisms proposed which suggest the divergence of Feynman series in theories with fermions. ${ }^{2,3}$ While it has certainly not been proven that fantastic cancelations do not occur in renormalizable but nonsuper-renormalizable series, ${ }^{4}$ it seems unlikely that perturbation theory converges in any Lagrangian field theory. On the other hand, much hard work has been done on proving properties of the Feynman perturbation series order by order. The most interesting properties are the nonlinear ones that do not hold exactly if the series is truncated to any fixed finite order, but only hold in the sense of formal power series. Among these are formal unitarity of the $S$ matrix ${ }^{5}$ and Ward identities, both nonanomalous and anomalous. ${ }^{6}$ It is our goal in this note to describe a mechanism which could make perturbative equations exact in an actual theory. We will use unitarity as an illustrative example-the same mechanism could operate in other cases. ${ }^{7}$

To put this mechanism in perspective, let us first discuss some simple summability techniques. In recent years, several techniques for summing divergent series have been proposed as computational devices ${ }^{8}$ or have been proven to be valid ${ }^{9}$ in relatives of the simplest of all divergent perturbation series: the ground-state energy of an anharmonic oscillator. ${ }^{10}$ Let us see how these summability techniques respect three types of conditions:

(i) Invariance conditions. - We have in mind properties like crossing symmetry in amplitudes with trivial crossing matrix or Lorentz invariance. These are clearly preserved automatically by any convergent summability method.

(ii) Linear covariances.-We have in mind crossing symmetry in amplitudes with nontrivial crossing matrices and Lorentz covariance for currents or other tensorial quantities. These are clearly preserved by linear summation procedures like Borel summability, but not automatically preserved by nonlinear summation processes like Padé summation.

(iii) Nonlinear perturbative properties, as mentioned above. - In general, these are not preserved by any summability methods although it is known that partial-wave unitarity in the elastic region is preserved by the diagonal Padé method. ${ }^{11}$ We thus see that a summability statement alone is not, in general, enough to carry formal computations from the power-series level to the "summed-series" level.

The mechanism which we wish to discuss has already been discussed in "physical situations" by the author elsewhere $\mathrm{e}^{3,12}$; it is implicit in the classical work of Watson and Carleman ${ }^{13}$ for formal asymptotic series. We say a function $f$ obeys a strong asymptotic condition (SAC) and has $\sum a_{n} z^{n}$ as a strong asymptotic series (SAS) if and only if (a) $f$ is analytic in a sectorial region $S$ of the form $\{z|| \arg z|<\theta, 0<| z \mid<B\}$ for some $\theta>\frac{1}{2} \pi$ and some $B>0$ (in field theories, one expects that $\theta$ can be chosen arbitrarily close to $\left.\pi^{9,3}\right)$; and (b) there exist constants $A$ and $\sigma$ so that for all $N$ and $z \in S$

$$
\left|f(z)-\sum_{n=0}^{N} a_{n} z^{n}\right| \leqslant A \sigma^{N+1}(N+1) !|z|^{N+1} .
$$

There are two critical properties of functions obeying a SAC:

(I) Any function $f$ with SAS whose coefficients are all 0 is identically $0 .-$ Equivalently, there is at most one function with any given SAS. This is just Carleman's theorem ${ }^{13}$ or follows alternately from the fact that $f$ can be recovered from $\sum a_{n} z^{n}$ by Borel summation. 
(II) Products of functions obeying a SAC obey a $S A C$. - So do sums and so do quotients $f / g$ if the lowest-order term in $g$ is nonzero. At first sight, one might expect that products have remainders growing like $[(N+1) !]^{2}$, not $(N+1)$ !. However, a simple proof shows that if $f$ has $\sum a_{n} z^{n}$ as a SAS and $g$ has $\sum b_{n} z^{n}$ as a SAS, then $f g$ has $\sum c_{n} z^{n}$ as a SAS with

$$
c_{n}=\sum_{m=0}^{n} a_{m} b_{n-m} \text {. }
$$

As a typical element of the proof let us show that $\left|c_{N}\right|<A \sigma^{N} N$ ! for all $N$. Since (1) holds for $f$ and g, $\left|a_{n}\right| \leqslant A_{1} \sigma_{1}{ }^{N} N !$ and $\left|b_{n}\right| \leqslant A_{2} \sigma_{2}{ }^{N} N$ !, then .

$$
\begin{aligned}
\left|c_{n}\right| & \leqslant \sum_{m=0}^{N}\left|a_{m}\right|\left|b_{N-m}\right| \\
& \leqslant A_{1} A_{2} \sum_{m=0}^{N}{\sigma_{1}}^{m} \sigma_{2}{ }^{N-m} m !(N-m) ! \\
& \leqslant N !\left(\sigma_{1}+\sigma_{2}\right)^{N}\left(A_{1} A_{2}\right),
\end{aligned}
$$

since $[m !(N-m) !]^{2}<(N !)^{2}$.

As a typical "nonlinear summation theorem" that follows from (I) and (II), we note the following:

Proposition.-Suppose at some fixed energy $E$, the re are $n<\infty$ open channels for $a+b$ scattering given by amplitudes $T_{1}\left(E ;\left\{p_{1}\right\}, g\right), \cdots, T_{n}\left(E ;\left\{p_{n}\right\}\right.$, $g)$, where $g$ is the coupling constant in some underlying field theory and $\left\{p_{i}\right\}$ is a set of kinematic variables for channel $i$. Suppose in some region

$$
S=\left\{g|0<| g|<G,| \arg g \mid<\theta ; \theta>\frac{1}{2} \pi\right\} ;
$$

the $T_{i}\left(E ;\left\{p_{i}\right\}, g\right)$ have the Feynman series for the process $a+b \rightarrow i$ as a SAS with the constants $\sigma, A$ in (1) bounded uniformly as the $\left\{p_{i}\right\}$ vary over their (compact) set of allowable phase space. Then forward unitarity holds at energy $E$ with 0 $<g<G$.

The proof of this proposition is trivial given (I) and (II); for unitarity says that

$$
\begin{aligned}
& \operatorname{Im} T_{\mathrm{e} 1}(0, g) \\
& \quad=\sum_{i=1}^{n} \int d \rho\left(p_{i}\right) T_{i}\left(p_{i}, g^{*}\right) * T_{i}\left(p_{i}, g\right) .
\end{aligned}
$$

Both sides of (2) are analytic functions obeying a SAC [using (II) and the hypothetical uniform estimates on $\sigma, A]$. By formal unitarity ${ }^{5}$ their SAS are equal, so by (1) they are equal.

Similar arguments hold for any other nonlinear relation. It is a difficult and open mathematical question $^{14}$ as to when there is any function with a given SAS, so we are far from using the ideas of this Letter in constructing quantum fields. Nevertheless, we have presented a simple mechanism that could validate formal perturbation theory arguments even when the series in question diverge.

*Research partially supported by the U. S. Air Force Office of Scientific Research under Contract No. F4462071-C-0108.

$\uparrow$ Alfred P. Sloan Foundation Fellow.

${ }^{1}$ A. Jaffe, Commun. Math. Phys. 1, 127 (1965); C. Hurst, Proc. Cambridge Phil. Soc. 48, 625 (1952); W. Thirring, Helv. Phys. Acta 26, 33 (1953); A. Peterman, Helv. Phys. Acta 26, 291 (1953).

${ }^{2}$ For quantum electrodynamics, see F. Dyson, Phys. Rev. 85, 631 (1952).

${ }^{3}$ For $Y_{2}$, a mechanism whereby renormalization counterterms cause a divergence is proposed by B. Simon, in the Coral Gables Conference on Fundamental Interactions at High Energy, University of Miami, Coral Gables, Florida, January 1972 (Gordon and Breach, New York, to be published). See also, F. Guerra and M. Mariano, Nuovo Cimento 42A, 285 (1966).

${ }^{4}$ In fact, the indications are that renormalization makes convergence worse; see B. Simon, Nuovo Cimento 59A, 166 (1969).

${ }^{5} \mathrm{~K}$. Hepp, in Field Theory and Statistical Mechanics: 1970 Les Houches Lectures, edited by R. Stora and C. DeWitt (Gordon and Breach, New York, 1972); H. Epstein and V. Glaser, ibid.

${ }^{6}$ Nonanomalous: see, e.g., J. Ward, Phys. Rev. 78, 1824 (1950); Anomalous: see, e.g., S. Adler, Phys. Rev. 177, 2426 (1969).

${ }^{7}$ More complex arguments such as the derivation of the Callan-Symanzik relations [K. Symanzik, Commun. Math. Phys. 18, 227 (1970); G. Callan, Phys. Rev. D 2 , 1541 (1970)], which rely on Weinberg's theorem and would require Eq. (1) to hold uniformly as certain variables approached infinity, are more dubious from our point of view.

${ }^{8}$ The Pade method was proposed in L. Copley and D. Masson, Phys. Rev. 164, 2059 (1967); D. Bessis and L. Pusterla, Nuovo Cimento 60A, 243 (1969).

${ }^{9}$ For Pade, see J. J. Loeffel et al., Phys. Lett. 30B, 656 (1969); for Borel summability, see S. Graffi et al., Phys. Lett. 32B, 631 (1970); B. Simon, Phys. Rev. Lett. 25, 1583 (1970); for Euler summability, see $\mathrm{J}$. Gunson and D. $\mathrm{Ng}$, to be published.

${ }^{10} \mathrm{C}$. Bender and T. T. Wu, Phys. Rev. 184, 1231 (1969); B. Simon, Ann. Phys. (New York) 58, 79 (1970).

${ }^{11}$ This is most thoroughly discussed in J. Basdevant et al., Nuovo Cimento 60A, 185 (1969).

${ }^{12}$ B. Simon, Advan. Math. 7,240 (1971).

${ }^{13} \mathrm{G}$. Watson, Phil. Trans. Roy. Soc. London, Ser. A 211, 279 (1912); T. Carleman, Les Fonctions Quasianalytiques (Gauthier-Villars, Paris, 1926).

${ }^{14}$ For example, $\sum(-1)^{n} n ! z^{n}$ is a SAS for a some function, while $\sum n ! z^{n}$ and $\left.\sum n !\left(-2^{n+1}\right)+1\right]$ are not the SAS for any function. 